\title{
Borderline Intermediary Thickness Cutaneous Melanoma: Standard Approach and The New Possibilities for Removal in one Surgical Session
}

\author{
Oliveira $\mathbf{N}^{1}$, Kandathil LJ ${ }^{1}$, Tchernev $\mathrm{G}^{2^{*}}$ \\ ${ }^{1}$ Medical Student, Faculty of Medicine, Medical University of Sofia, Bulgaria \\ ${ }^{2}$ Onkoderma - Clinic for Dermatology, Venereology and Dermatologic Surgery, Sofia, Bulgaria
}

Received: May 25, 2021; Accepted: May 29, 2021; Published: June 01, 2021

*Corresponding author: GeorgiTchernev, Onkoderma-Clinic for Dermatology, Venereology and Dermatologic Surgery, General Skobelev, Email: georgi_tchernev@yahoo.de

\section{Case Report}

Here we present a case of a 48-year-old female with a melanocytic lesion on the lateral aspect of the right thigh [Figure $1 \mathrm{a}-1 \mathrm{~d}]$. The patient observed a steady growth of the lesion over the last few years and attended our clinic for a dermatological consultation in March 2021. She was otherwise healthy and she reported anamnestic data was unremarkable. On examination of the lateral femoral region of the right thigh, the presence of a single, nodular, hyperpigmented lesion of $1.5 \mathrm{~cm}$ was noted. The dome shaped lesion was dark brown in colour, smooth and elevated on palpation, and well demarcated from surrounding healthy tissue. Based on the clinical and dermatoscopical findings, a nodular melanoma of intermediate thickness was suspected. Further laboratory workup was conducted and showed no significant deviations from normal paraclinical values. Screening was also negative for tumour spread. All other paraclinical data was unremarkable. Diagnostic work up including CT of the thorax and abdomen detected no metastatic dissemination. The standard two step approaches based on the current AJCC guidelines was employed. The primary excision was performed with $0.5 \mathrm{~cm}$ safety margins in all directions [Figure 1e-f]. The elliptical defect was subsequently closed by single interrupted sutures. Histopathological verification confirmed the diagnosis of a nodular malignant melanoma, pT2b N0M0, stage 1B, Clark IV, Breslow thickness of $2 \mathrm{~mm}$, without ulceration, with high mitotic activity, well defined lymphocytic stromal reaction and clean resection margins.

The patient was sent to the National Oncology Hospital for a secondary re-excision and sentinel lymph node biopsy (SLNB) staging a week later. Re-excision of $1 \mathrm{~cm}$ from the previous cicatrix was carried out alongside drainage of SLNB at the right inguinal region [Figure $2 \mathrm{c}$-d] which was negative for metastatic spread. After successful closure of the secondary re-excision [Figure 2a-b] and drainage [2c-d], the patient reported an excelled post-operative follow up a month later with no further signs of complications.

The current AJCC guideline defines an intermediate thickness melanoma of 1-4mm to undergo a two-step surgical approach, as the most effective treatment plan[1][Table 1]. Based on the established guidelines a primary excision is always conducted with a surgical margin of no more than $0.5 \mathrm{~cm}$ in all directions, followed by histopathological verification of tumour thickness. A secondary re-excision is then employed with a variable margin of $0.5-1.5 \mathrm{~cm}$ (AJCC recommendations) with or without SLNB [1]. The arbitrary decision defining the safety margins for the secondary re-excision is left to the choice of the clinician and although, is made based on the tumour depth, is not accurately specified (AJCC recommendations), leaving room for some confusion, especially for patients with intermediary thickness melanomas between 1 and $2 \mathrm{~mm}$ tumour thickness [Table 1] [3]. One Step Melanoma Surgery (OSMS) aims to clarify this uncertainty and provides a much clearer and precise approach to melanoma treatment in all stages but especially for thin and intermediary thickness cutaneous melanomas [Table 2] [2-3].

In our presenting case of nodular malignant melanoma, implementing personalised melanoma surgery in the face of OSMS would provide a favourable outcome based on the patient's clinical and dermatoscopic findings [2-3].

This would be achieved by carrying out a possible preoperative ultrasound to assess tumour thickness and perform a single excision with a $1 \mathrm{~cm}$ surgical margin alongside SLNB[Table 2]. A comprehensive approach such as this would achieve the final intended result of AJCC- all in one session[2-4]. The benefits of One step melanoma surgery/ OSMS treatment for melanoma are: 1) minimising psycho-emotional stress that comes from two surgical interventions [3]; 2) less room for surgical error and side effects [4]; 3)relieving patients from financial burden[2]; 4) reduced risk of patients not willing to appear for the second procedure due to fear $[2,4]$.

Although we present a case that followed the AJCC recommendations, we propose the possibility of using the novel innovative OSMS approach. This guideline encompasses all the necessary requirements in achieving the optimal treatment outcome in one session. 

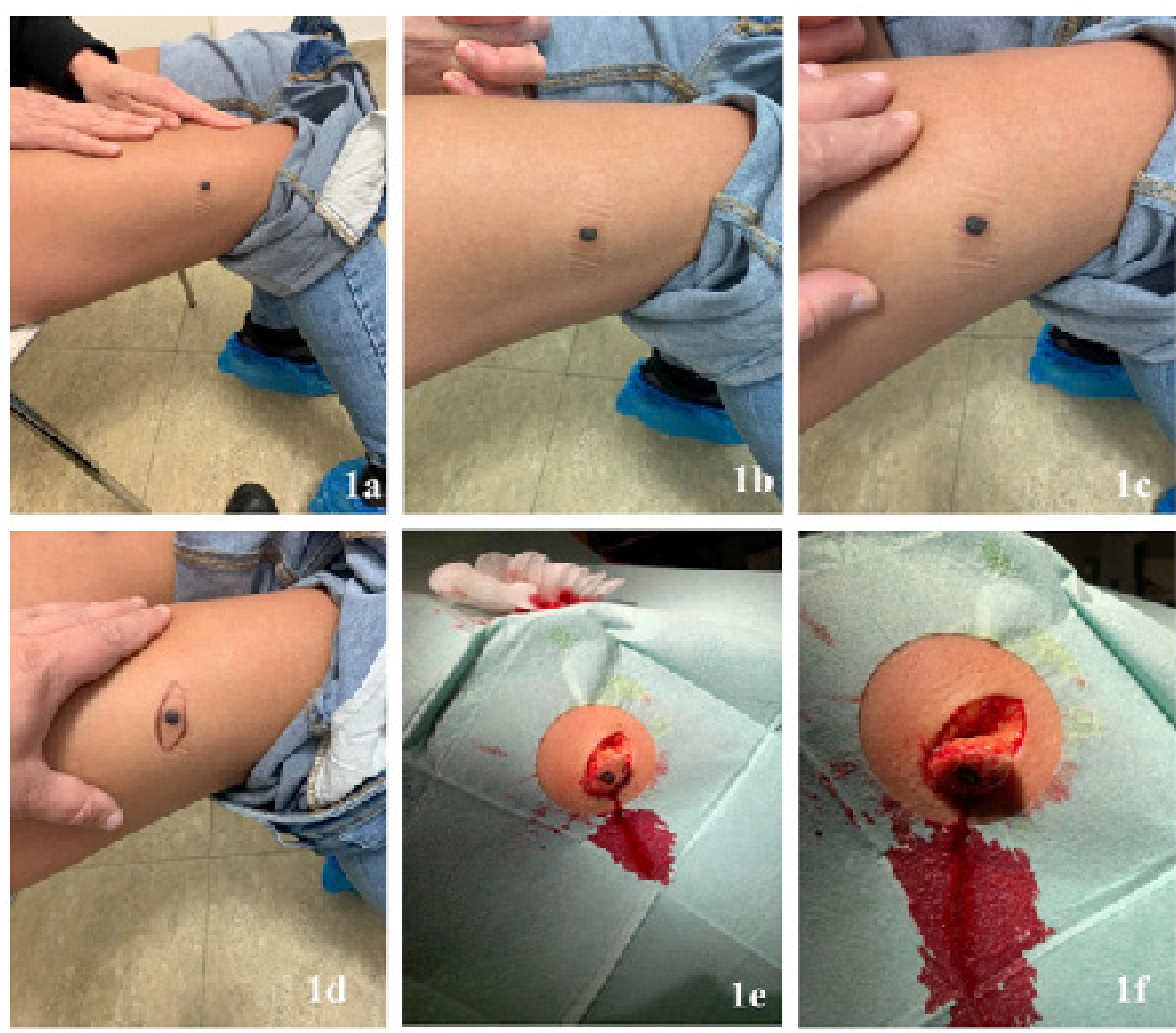

Fig 1a-c: Hyperpigmented melanocytic nodule of $1.5 \mathrm{~cm}$ diameter localised to the right outer thigh region. Lesion well demarcated from healthy tissue.

Fig 1d: Preoperative outlining of the surgical safety margins of $0.5 \mathrm{~cm}$ in all directions according to AJCC guidelines for primary excision.

Fig 1e-f: Intraoperative finding with elliptical excision of the defect \& removal of the lesion 

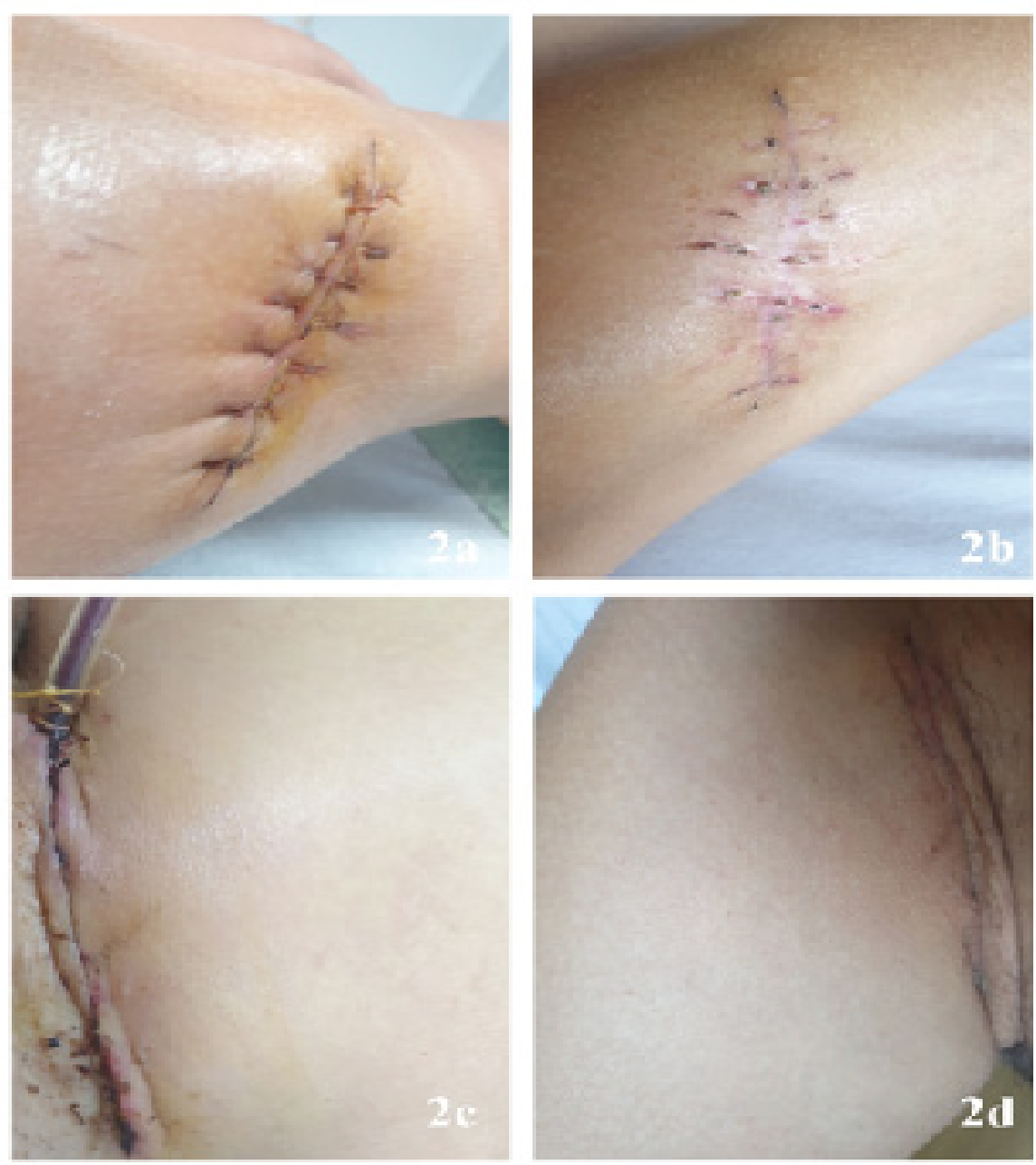

Fig 2a: Postoperative finding after secondary re-excision: Final closure of the surgical defect with 7 single interrupted sutures.

Fig 2b: Clinical picture during follow up assessment of the secondary re-excision. Good postoperative finding after suture removal with good healing of cicatrix

Fig 2c: Drainage of the sentinel lymph node in the right inguinal region.

Fig 2d: Postoperative picture after drainage of sentinel lymph node.

\begin{tabular}{|c|c|}
\hline \multicolumn{2}{|l|}{ Table I : AJCC recommandations (Swetter et al., 2019) } \\
\hline Breslow thickness & Recommended surgical margins / AJCC \\
\hline Melanoma in situ & $0.5 \mathrm{~cm}$ (primary excision with $0.5 \mathrm{~cm}$ in all directions, followed by secondary excision) \\
\hline$<1 \mathrm{~mm}$ & $0.5 \mathrm{~cm}$ primary excision (followed by secondary excision with $0.5 \mathrm{~cm}$ in all directions) \\
\hline $1.01-2.0 \mathrm{~mm}$ & $\begin{array}{c}0.5 \mathrm{~cm} \text { primary excision (followed by secondary excision with } 0.5-1.5 \mathrm{~cm} / \text { complete surgical field } \\
\text { between } 1 / 2 \mathrm{~cm} \text { with SLNB) }\end{array}$ \\
\hline $2 \mathrm{~mm}-4 \mathrm{~mm}$ & $0.5 \mathrm{~cm}$ primary excision (followed by secondary with $1.5 \mathrm{~cm}$ in all directions/ with SLNB) \\
\hline$>4 \mathrm{~mm}$ & $\begin{array}{c}0.5 \mathrm{~cm} \text { primary excision (followed by secondary excision with } 1.5 \mathrm{~cm} \text { in all directions/ without } \\
\text { SLNB if nodes not enlarged/matter of discussion) }\end{array}$ \\
\hline
\end{tabular}


Borderline Intermediary Thickness Melanoma: Standard Approach and the New Possibilities for Removal in one Surgical Session

\begin{tabular}{|c|c|}
\hline Breslow thickness & Recommended surgical margins/ Tchernev et al. \\
\hline Melanoma in situ & $\begin{array}{l}1.0 \mathrm{~cm} \text { (clinical/dermatoscopic evaluation obligate/ if possibility for echographical examination- } \\
\text { from benefit) }\end{array}$ \\
\hline$<1 \mathrm{~mm}$ & $\begin{array}{l}1.0 \mathrm{~cm} \text { (clinical/dermatoscopic evaluation obligate/ if possibility for echographical examination- } \\
\text { from benefit) }\end{array}$ \\
\hline $1.01-2.0 \mathrm{~mm}$ & $1.0 \mathrm{~cm}$ (with SLNB), (echographic tumour thickness measurement preoperatively) \\
\hline $2 \mathrm{~mm}-4 \mathrm{~mm}$ & $2.0 \mathrm{~cm}$ (with SLNB), (echographic tumour thickness measurement preoperatively) \\
\hline$>4 \mathrm{~mm}$ & $\begin{array}{l}2.0 \mathrm{~cm} \\
\text { a. If no enlarged lymph nodes- } 2 \mathrm{~cm} \text { resection is sufficient } \\
\text { a. Presence of enlarged lymph nodes- to be removed together with } \\
\text { the re excision of primary lesion! }\end{array}$ \\
\hline
\end{tabular}

\section{References}

1. Swetter S, Tsao H, Bichakjian C, Lewandrowski C, Elder D, Gershenwald J, et al. Guidelines of care for the management of primary cutaneous melanoma. J Am Acad Dermatol. 2019;80(1):208-250.

2. Tchernev G, Temelkova I. Multiple Primary Cutaneous Melanomas in a Bulgarian Patient: The Possible Role of One Step Melanoma Surgery (OSMS) As the Most Adequate Treatment Approach! Open Access Maced J Med Sci. 2018;6(11):2155-2160. doi:10.3889/ oamjms.2018.487

3. Tchernev G. One Step Surgery for Cutaneous Melanoma: "We Cannot Solve Our Problems with the Same Thinking We Used When We Created Them?" Open Access Maced J Med Sci. 2017;5(6):774-776. doi: 10.3889/oamjms.2017.168

4. Tchernev G. Novel Surgical Approach in Cutaneous Melanoma Patients: "Daring Ideas Are Like Chessmen Moved For- ward. They May Be Beaten, But they May Start a Winning Game!?" Open Access Maced J Med Sci. 2017;5(6):810-812. doi:10.3889/oamjms.2017.199 